\title{
Gyrostatic extensions of the Howard-Krishnamurti model of thermal convection with shear
}

\author{
C. Tong ${ }^{1}$ and A. Gluhovsky ${ }^{2}$ \\ ${ }^{1}$ Biometrics Research RY33-300, Merck Research Laboratories, P.O.Box 2000, Rahway, NJ, 07065, USA \\ ${ }^{2}$ Department of Earth and Atmospheric Sciences and Department of Statistics, Purdue University, 550 Stadium Mall Drive, \\ West Lafayette, IN, 47907-2051, USA
}

Received: 31 July 2007 - Revised: 18 October 2007 - Accepted: 3 January 2008 - Published: 6 February 2008

\begin{abstract}
The Howard \& Krishnamurti (1986) low-order model (LOM) of Rayleigh-Bénard convection with spontaneous vertical shear can be extended to incorporate various additional physical effects, such as externally forced vertical shear and magnetic field. Designing such extended LOMs so that their mathematical structure is isomorphic to those of systems of coupled gyrostats, with damping and forcing, allows for a modular approach while respecting conservation laws. Energy conservation (in the limit of no damping and forcing) prevents solutions that diverge to infinity, which are present in the original Howard \& Krishnamurti LOM. The first LOM developed here (as a candidate model of transverse rolls) involves adding a new Couette mode to represent externally forced vertical shear. The second LOM is a modification of the Lantz (1995) model for magnetoconvection with shear. The modification eliminates an invariant manifold in the original model that leads to potentially unphysical behavior, namely solutions that diverge to infinity, in violation of energy conservation. This paper reports the first extension of the coupled gyrostats modeling framework to incorporate externally forced vertical shear and magnetoconvection with shear. Its aim is to demonstrate better model building techniques that avoid pathologies present in earlier models; consequently we do not focus here on analysis of dynamics or model validation.
\end{abstract}

\section{Introduction}

Rayleigh-Bénard convection is the buoyancy-driven circulation in a shallow layer of Newtonian fluid contained between two horizontal, isothermal surfaces, the lower one at higher temperature than the upper one, in the presence of a constant, uniform, vertical gravitational field (Chandrasekhar, 1961;

Correspondence to: $\mathrm{C}$. Tong

(ctong@mailaps.org)
Koschmieder, 1993; Getling, 1998; Mutabazi et al., 2006). The response of the fluid to the imposed, adverse temperature gradient depends, in part, on the Rayleigh number, $R a$, a nondimensional control parameter assessing the relative strength of buoyancy to dissipative effects. Rayleigh-Bénard convection is a classical problem in hydrodynamic instability, with applications in geophysics and astrophysics as well as in various industrial processes. For instance, RayleighBénard convection serves as a model system for both mantle convection in geophysics (Peltier, 1989) and mesoscale shallow convection in atmospheric boundary layers (Brown, 1980; Agee, 1987; Atkinson and Zhang, 1996). Furthermore, thermal convection of an electrically conducting fluid in the presence of a magnetic field is a model system for magnetoconvection in the solar photosphere (Proctor and Weiss, 1982).

Over twenty-five years ago, Krishnamurti and Howard (1981) discovered that spontaneous vertical shear could be generated in a Rayleigh-Bénard convection laboratory experiment, resulting in large scale horizontal flow coexisting with local convective structures. The first theoretical evidence reinforcing the possibility of such large scale circulations was provided by their study of a low-order model (Howard and Krishnamurti, 1986), which has since been widely used and extended. A low-order model (LOM) is a nonlinear dynamical system usually derived from a severely truncated spectral Galerkin approximation (Shirer, 1987; Holmes et al., 1996). The hydrodynamic fields (in this case, streamfunction and temperature) are expanded in infinite series of time-independent, orthogonal eigenfunctions that satisfy the boundary conditions. The series are then truncated, usually arbitrarily, and the remaining finite set of time-dependent "Fourier" amplitudes satisfy a set of coupled, nonlinear ordinary differential equations (the LOM).

The purpose of a low-order model is usually not to have a numerically accurate computational fluid dynamics (CFD) simulation of the flow. Rather, LOMs provide a simplified,

Published by Copernicus Publications on behalf of the European Geosciences Union and the American Geophysical Union. 
abstract representation of the physical situation that may help understand a critical feature of the dynamics, such as the sensitive dependence on initial conditions revealed by the Lorenz (1963) model or the theoretical plausibility of large scale circulation in the Howard and Krishnamurti (1986) model. The latter model is not intended to be an exact description of large scale circulation in thermal convection, but rather a plausibility argument that such circulations can be exhibited in a theoretical model that represents, at some level of abstraction, the Rayleigh-Bénard flow.

Howard and Krishnamurti (1986) noted that, due to a deficiency in the selection of modes retained in the truncation, their LOM possesses an invariant manifold on which the system is linear and unstable at sufficiently high Rayleigh number, resulting in trajectories that diverge to infinity. Thiffeault and Horton (1996) showed that the Howard-Krishnamurti (HK) model could be extended by adding one mode to the temperature expansion, so that conservation of energy (in the dissipationless limit) is restored. The resulting LOM has neither the offending invariant manifold nor solutions diverging to infinity; instead, it exhibits better agreement with experimental heat flux data than the original HK model (Thiffeault and Horton, 1996). Meanwhile, Hermiz et al. (1995) showed that, in order to satisfy a vorticity balance condition derived by Lantz (1995), the HK model should include an additional mode in the streamfunction expansion.

Indeed, the Galerkin technique for deriving LOMs does not provide any guidance on the selection of modes to retain in the truncation (Treve and Manley, 1982), and careless choices can lead to the potential for unphysical behavior such as solutions that diverge to infinity. A physicallymotivated, modular approach to designing LOMs using the Galerkin method has been introduced (Gluhovsky, 1982, 1986; Gluhovsky and Tong, 1999; Gluhovsky et al., 2002; Gluhovsky, 2006). This approach is based on designing LOMs so that their mathematical equations have a structure isomorphic to those for systems known in mechanics as coupled gyrostats, with friction and forcing. In mechanics, a gyrostat is a system of bodies whose relative motion cannot alter the intrinsic mass distribution of the system; the principal example is an arbitrarily shaped rigid body, the carrier, with an axisymmetric rotor that rotates (with constant angular velocity) about an axis fixed in the carrier body (e.g. Gluhovsky and Tong, 1999).

In rescaled variables, the Volterra (1899) equations of motion for a gyrostat have the following form:

$$
\begin{aligned}
& \dot{v}_{1}=p v_{2} v_{3}+b v_{3}-c v_{2}, \\
& \dot{v}_{2}=q v_{3} v_{1}+c v_{1}-a v_{3}, \\
& \dot{v}_{3}=r v_{1} v_{2}+a v_{2}-b v_{1},
\end{aligned}
$$

where $a, b, c, p, q$, and $r$ are constants characterizing the geometry of the gyrostat, with the constraint that

$p+q+r=0$, and $v_{1}, v_{2}$, and $v_{3}$ are state variables that describe the dynamics of the system (Gluhovsky, 1982; Gluhovsky and Tong, 1999). This system is quadratically nonlinear, like the Navier-Stokes equations, and it possesses two quadratic integrals of motion and also conserves phase space volume. Damping and driving terms may be added to Eq. (1). If linear viscous friction and constant forcing are used, the solutions are guaranteed to be bounded (Gluhovsky and Tong, 1999).

The Volterra gyrostat can be used as a "building block" for building higher order dynamical systems, by coupling together multiple gyrostats. Such systems maintain the conservation properties of interest. For instance, any system of coupled gyrostats conserves the sum of squares of all dynamic variables, a quantity usually representing some form of energy. An increase in the order of approximation of a LOM may be carried out by adding gyrostat modules. The modules' internal coefficients can be modified to represent adding various physical effects, such as rotation, topography, and magnetic field (Gluhovsky and Agee, 1997; Gluhovsky and Tong, 1999). This is because the gyrostats that appear in LOMs are usually not of the general form (1) but rather one of its special cases. For instance, the widely-known Lorenz (1963) model of Rayleigh-Bénard convection has the form of (1) but with $r=b=c=0$ and with additional friction and forcing terms, as can be seen after a linear change of variables (Gluhovsky, 1982). This version of the gyrostat will be referred to as the Lorenz gyrostat. Another gyrostat commonly encountered in LOMs is the Euler gyroscope, where $a=b=c=0$ so that only the three nonlinear terms are present (Obukhov, 1969). For a thorough discussion of Eq. (1), including the relationships between the fluid dynamical and rigid bodies interpretations of the gyrostat, see Gluhovsky and Tong (1999). For a discussion of the general relationships between systems of coupled gyrostats, including their generalizations that incorporate nonlinear feedback, and energy-conserving low-order models, see the work of Lakshmivarahan and Wang (2008a,b). In particular, they establish the equivalence between a class of energy-conserving LOMs characteristic of magnetohydrodynamics and systems of coupled gyrostats, and provide algorithms for converting such LOMs into coupled gyrostat form.

In previous work, we showed that the Thiffeault and Horton (1996) LOM has the form of four coupled gyrostats, with friction and forcing, and demonstrated that it possesses two integrals of motion corresponding to conservation of total mechanical energy and of "unavailable" energy (Gluhovsky and Tong, 1999). Subsequently, we added the streamfunction mode of Hermiz et al. (1995) to the Thiffeault-Horton LOM, which results in a system of six coupled gyrostats that respects both energy and vorticity balance (Gluhovsky et al., 2002). In this paper, we expand the scope of the coupled gyrostats approach for designing LOMs by showing how the HK model can be further extended to include (1) a representation of externally forced shear, by extending our previous LOM (Gluhovsky et al., 2002), and (2) magnetoconvection 
with shear, by extending the LOM of Lantz (1995). These will be discussed in the next two sections, respectively. (Neither physical mechanism was previously known to be within the scope of applicability of the coupled gyrostats approach.) A concluding section will explore additional implications beyond the HK model. (An appendix presents an informal approach for converting a LOM into a system of coupled gyrostats, when this is possible.)

Since low-order models continue to be used recently for the investigation of various flow phenomena, e.g. Fütterer (2003); Chen and Price (2004); Moehlis (2004); Lakshmivarahan et al. (2006); Chen and Price (2006); Peng et al. (2007), and Roy and Musielak (2007), there remains a need to foster innovative principles for designing LOMs such as those discussed here. This paper limits its scope to the contribution of coupled gyrostats to low-order model building. In this paper we do not discuss other key issues in mathematical modeling, such as convergence and validation, the investigation of model dynamics, and the comparison of model results with observational or experimental data. Of course, these latter issues should be considered when deploying coupled gyrostat LOMs to physical problems. We also do not claim to provide a general recipe for LOM construction with a fail-safe procedure for picking the "right" modes. Rather, we show how to improve existing practices of often arbitrary mode selection, which can result in unphysical behavior. We focus on illustrating how our technique can assist in model building by enforcing conservation properties and boundedness of solutions in the presence of novel physical mechanisms (externally forced shear and magnetoconvection with shear). Our approach certainly eliminates the most extreme pathology of unfettered model building: solutions that diverge to infinity. There are no parameter regimes in which coupled gyrostat LOMs exhibit such pathologies.

\section{Externally forced shear}

The problem of Rayleigh-Bénard convection in an externally sheared flow has numerous geophysical and engineering analogs (Kelly, 1994), for instance, in the formation of cloud rows in a convective atmospheric boundary layer (Brown, 1980; Shirer, 1986; Atkinson and Zhang, 1996). Usually convection rolls with axes parallel to the shear direction are found, e.g. in experiments and in meteorological observations of cloud bands. These longitudinal rolls are preferred when the basic Couette flow loses stability due to the thermal stress. However, transverse rolls, whose axes are perpendicular to the direction of shear, are known to occur in certain situations, such as moderately supercritical convection with very low Reynolds number in air (Graham, 1933; Chandra, 1938; Bénard and Avsec, 1938). For more discussion of experimental work on transverse rolls, see the reviews by Brunt (1951) and Kelly (1994), and the paper by Ingersoll
(1966). The LOM to be developed below is proposed as a model of transverse rolls.

The basic momentum and heat equations for twodimensional Rayleigh-Bénard convection can be written in the following nondimensional way (Saltzman, 1962; Howard and Krishnamurti, 1986):

$$
\begin{aligned}
\frac{\partial}{\partial t} \nabla^{2} \psi+\frac{\partial \psi}{\partial z} \frac{\partial}{\partial x} \nabla^{2} \psi-\frac{\partial \psi}{\partial x} \frac{\partial}{\partial z} \nabla^{2} \psi & =\operatorname{Pr} \frac{\partial \theta}{\partial x} \\
& +\operatorname{Pr} \nabla^{4} \psi, \\
\frac{\partial \theta}{\partial t}+\frac{\partial \psi}{\partial z} \frac{\partial \theta}{\partial x}-\frac{\partial \psi}{\partial x} \frac{\partial \theta}{\partial z} & =\operatorname{Ra} \frac{\partial \psi}{\partial x}+\nabla^{2} \theta,
\end{aligned}
$$

where $\psi$ is the streamfunction, $\theta$ is the temperature deviation from the conductive steady-state profile, $\mathrm{Pr}$ is the Prandtl number, and $R a$ is the Rayleigh number. Here, $t$ is time, $x$ is position along the horizontal axis, and $z$ is position along the vertical axis.

The boundary conditions at the top and bottom of the layer are that $\theta \equiv \partial \psi / \partial x \equiv \partial^{2} \psi / \partial z^{2} \equiv 0$ at $z=0, \pi$, which includes the stress-free condition. (However, note that any externally-forced shear mechanism could not be subject to the stress-free condition.) For a flow without vertical shear, periodic boundary conditions of the form, $\partial \psi / \partial z \equiv \partial \theta / \partial x \equiv 0$ at $x=0, \pi / a$, where $a$ is the aspect ratio (cell height to cell width), are often assumed. However, to allow for vertical shear, Howard and Krishnamurti (1986) and Thiffeault and Horton (1996) do not impose these periodic boundary conditions and deliberately include Fourier modes that do not obey them. Rather, in the Galerkin expansions they simply choose to seek solutions that have horizontal wavenumber $a$, but include modes that are $x$-independent, without which there would be no large scale circulation (which they wish to study). We shall follow their approach here.

Using the following Galerkin expansions,

$$
\begin{aligned}
\psi(x, z, t)=A( & t) \sin (a x) \sin (z)+B(t) \sin (z) \\
& +C(t) \cos (a x) \sin (2 z)+G(t) \sin (3 z) \\
& +\frac{\pi}{2} \epsilon z^{2}, \\
\theta(x, z, t)=D(t) \cos (a x) \sin (z)+E(t) \sin (2 z) & +F(t) \sin (a x) \sin (2 z)+H(t) \sin (4 z),
\end{aligned}
$$

the following LOM may be derived:

$$
\begin{array}{r}
\dot{A}=-\operatorname{Pr}\left(1+a^{2}\right) A+\frac{a P r}{1+a^{2}} D+\frac{a}{2}\left(\frac{3+a^{2}}{1+a^{2}}\right) B C \\
+\frac{3 a}{2}\left(\frac{5-a^{2}}{1+a^{2}}\right) C G-\frac{16 a}{9}\left(\frac{4+a^{2}}{1+a^{2}}\right) \epsilon C, \\
\dot{B}=-\operatorname{Pr} B-\frac{3 a}{4} A C, \\
\dot{C}=-\operatorname{Pr}\left(4+a^{2}\right) C-\frac{a P r}{4+a^{2}} F-\frac{a^{3}}{2\left(4+a^{2}\right)} A B \\
-\frac{3 a}{2}\left(\frac{8-a^{2}}{4+a^{2}}\right) A G+\frac{16 a}{9}\left(\frac{1+a^{2}}{4+a^{2}}\right) \epsilon A,
\end{array}
$$




$$
\begin{aligned}
\dot{D}=-\left(1+a^{2}\right) D+a R a A-a A E-\frac{a}{2} B F \\
\quad+\frac{3 a}{2} F G+\frac{16 a}{9} \epsilon F \\
\dot{E}=-4 E+\frac{a}{2} A D, \\
\dot{F}=-\left(4+a^{2}\right) F-a \operatorname{RaC}+\frac{a}{2} B D+2 a C H \\
\quad-\frac{3 a}{2} D G-\frac{16 a}{9} \epsilon D, \\
\dot{G}=-9 P r G+\frac{a}{4} A C, \\
\dot{H}=-16 H-a C F .
\end{aligned}
$$

A version of this system, but with $\epsilon=0$, was studied previously by Gluhovsky et al. (2002). Without variables $G$ and $H$ and with $\epsilon=0$, the above system reduces to that of Howard and Krishnamurti (1986). The role of $H$ is to maintain conservation of energy (Thiffeault and Horton, 1996); the role of $G$ is to maintain conservation of vorticity (Hermiz et al., 1995). The new term in the streamfunction, proportional to $\epsilon z^{2}$, represents a linear shearing velocity profile superimposed onto the basic flow. This "Couette mode" is a crude representation of a fully developed, externally forced shear. Here, the shear is applied transversely to the axes of the rolls. The Reynolds number of the external shear mode can be expressed as $\operatorname{Re}=\pi^{2} \epsilon / \operatorname{Pr}$.

Strictly speaking, the $\epsilon z^{2}$ term in the streamfunction expansion is not part of the Galerkin approximation, since it does not belong to the Fourier set of orthogonal eigenfunctions. Note that its magnitude is fixed as a parameter, $\epsilon$, while the magnitudes of the Fourier modes are time-dependent unknowns. Thus the externally forced shear term is treated as specified, and we apply the Galerkin expansion only to the unspecified component of the flow. Note also that the forced shear term does not satisfy the stress-free boundary condition, $\partial^{2} \psi / \partial z^{2} \equiv 0$, at the top and bottom of the layer; rather, it plays the role of a forcing mechanism not unlike the classical, laminar Couette parallel-plate flow. The stressfree boundary condition is imposed only on the unspecified component of the flow.

A linear change of variables is often required to show explicitly that a LOM has a coupled gyrostats form. The choice of transformation is usually not unique (for further discussion, see the Appendix). To give one example, the following transformation of variables,

$$
\begin{aligned}
& x_{1}=1+\frac{a^{2} \operatorname{Pr}}{16\left(1+a^{2}\right)}(\operatorname{Ra}-E), \\
& x_{2}=\frac{a^{2} \operatorname{Pr}}{16\left(1+a^{2}\right) \sqrt{2}} D \\
& x_{3}=\frac{a}{4 \sqrt{2}} A \\
& x_{4}=\frac{a}{4 \sqrt{2}}\left(\frac{4+a^{2}}{1+a^{2}}\right) C
\end{aligned}
$$

$$
\begin{aligned}
& x_{5}=\frac{a}{4\left(1+a^{2}\right)} B, \\
& x_{6}=\frac{a^{2} P r}{16\left(1+a^{2}\right) \sqrt{2}} F, \\
& x_{7}=\frac{4+a^{2}}{2\left(1+a^{2}\right)}+\frac{a^{2} \operatorname{Pr}}{16\left(1+a^{2}\right)}\left(\frac{R a}{2}-H\right), \\
& x_{8}=\frac{9 a}{4\left(1+a^{2}\right)} G,
\end{aligned}
$$

and where time is scaled up by a factor of four, transforms the above LOM into coupled gyrostat form, shown in Table 1 . In the Table, $\alpha_{2}=1 / b, \alpha_{3}=\alpha_{2} \operatorname{Pr}, \alpha_{4}=(3 / 4+1 / b) \operatorname{Pr}, \alpha_{5}=\operatorname{Pr} / 4$, $\alpha_{6}=\alpha_{4} / \operatorname{Pr}, \quad \alpha_{7}=4, \quad \alpha_{8}=9 \alpha_{5}, f_{1}=1+\tilde{r} \operatorname{Pr} / b^{2}, \quad f_{2}=2((4+$ $\left.\left.a^{2}\right) /\left(1+a^{2}\right)\right)+\tilde{r} \operatorname{Pr} / b^{2}, q=-a^{2} / 2, r=-1 /(2(1+4 /(3 b)))$, $p=-(q+r), Q=2 /(3 b)-3 / 2, R=a /(6+8 / b), \quad P=-$ $(Q+R), c=2 / b, d=2\left(1+a^{2}\right) /\left(4+a^{2}\right), b=4 /\left(1+a^{2}\right)$, and $\tilde{r}=R a / R a_{c}$, where $R a_{c}=\left(1+a^{2}\right)^{3} / a^{2}$ is the critical Rayleigh number at which the conductive steady state loses stability, under conditions of no external shear. Finally, $\epsilon^{\prime}=16 a \epsilon / 9$.

In Table 1, friction and (constant) forcing terms are to the immediate left of the first vertical bar. On the right of the first vertical bar, there are (consecutively) (I) a Lorenz gyrostat $\left(x_{1}, x_{2}, x_{3}\right)$, (II) an Euler gyroscope $\left(x_{3}, x_{4}, x_{5}\right)$ with a pair of linear terms added, thus becoming a gyrostat, (III) a degenerative gyrostat $\left(x_{2}, x_{5}, x_{6}\right)$ (Gluhovsky and Tong, 1999), (IV) another Lorenz gyrostat $\left(x_{4}, x_{6}, x_{7}\right)$, (V) a plain Euler gyroscope $\left(x_{3}, x_{4}, x_{8}\right)$, and finally (VI) a degenerative gyroscope $\left(x_{2}, x_{6}, x_{8}\right)$ (Gluhovsky and Tong, 1999).

This system of six coupled gyrostats differs from that studied in Gluhovsky et al. (2002) only by the terms with coefficient $\epsilon^{\prime}$. It is thus evident that adding the effect of externally forced shear adds new linear (gyrostatic) terms to existing gyrostats in the model. In particular, gyrostat II, originally an Euler gyroscope, is now a gyrostat with three nonlinear terms and two linear terms; and gyrostat III, originally a degenerative gyroscope, is now a degenerative gyrostat.

As with any system of coupled gyrostats, one can verify that the sum of squares of all dynamical variables is a conserved quantity in the absence of forcing and dissipation. This quadratic integral of motion is associated with the total energy of the system, a linear combination of kinetic, gravitational potential, and available potential energies (Gluhovsky and Tong, 1999). In addition, this model also possesses the same linear integral of motion, $R x_{5}-r x_{8}$, representing total vorticity conservation, as in the model of Gluhovsky et al. (2002). Therefore all conservation properties are maintained and solutions will be bounded (Gluhovsky and Tong, 1999). We do not present here the explicit equivalence of these invariants with their hydrodynamic counterparts, since the nondimensionalization scheme used to write Eqs. (34) involves normalizing streamfunction and temperature by the viscosity and thermal diffusivity (Saltzman, 1962). To demonstrate the equivalence of the LOM invariants with their hydrodynamic counterparts, one would like to take these dissipation parameters to the zero limit to show conservation. 
Table 1. The LOM for convection with externally forced shear in coupled gyrostats form.

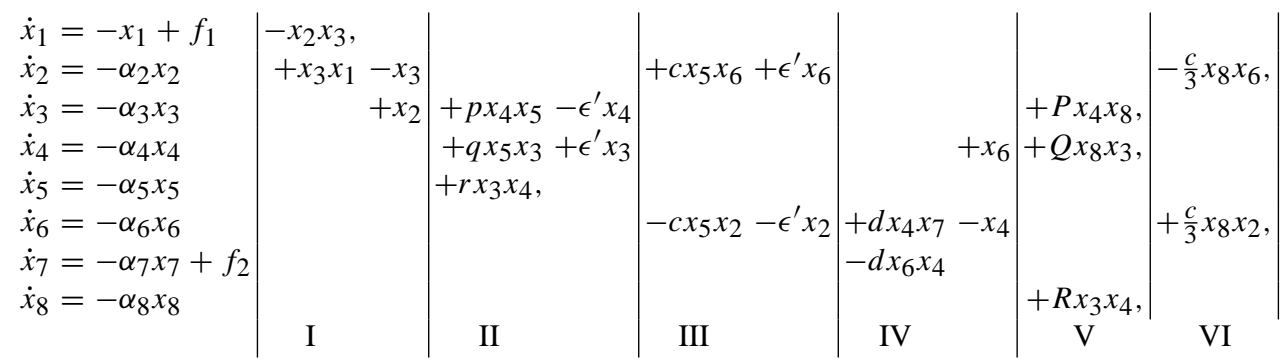

A more convenient but nonstandard nondimensionalization scheme would allow this to be done (see the example given by Tong and Gluhovsky (2002)).

The modular structure of this LOM is also worth attention. The addition of the externally forced shear mechanism results in modifying the existing gyrostatic modules in the LOM. Extending the order of approximation will result in adding new gyrostat modules to the system. For instance, adding two non-shear modes to the model, a $\sin (2 a x) \sin (z)$ mode in the streamfunction expansion and a $\cos (2 a x) \sin (z)$ mode in the temperature expansion, results in adding a new Lorenz gyrostat to the system. The new gyrostat couples the two new modes with the $E \sin (2 z)$ temperature mode. The new gyrostat does not involve the externally-forced shear parameter.

Note that the common modes selection procedure for LOMs is just to add a few more modes, the next ones in the Fourier expansion. We improve this by selecting only those among them that result in gyrostats, thereby maintaining energy conservation and bounded solutions. Our procedure can thus be used to assist in increasing the order of approximation of the Galerkin expansion. If one's goal is to formulate a numerically accurate CFD simulation, this also requires investigating the convergence of the Galerkin approximation as one continues to add modes (or gyrostat modules).

\section{Magnetoconvection with shear}

Lantz (1995) extended the HK model to the case of 2$\mathrm{D}$ magnetoconvection with an imposed horizontal magnetic field. His aim was to model magnetoconvection with spontaneously generated vertical shear, a phenomenon originally observed in a numerical simulation of the magnetoconvection partial differential equations (Lantz and Sudan, 1995). The basic momentum, heat, and magnetic flux equations, in nondimensional form, are as follows (Lantz, 1995):

$$
\begin{aligned}
\frac{\partial}{\partial t} \nabla^{2} \psi & +\frac{\partial \psi}{\partial z} \frac{\partial}{\partial x} \nabla^{2} \psi-\frac{\partial \psi}{\partial x} \frac{\partial}{\partial z} \nabla^{2} \psi \\
= & \operatorname{Pr} \frac{\partial \theta}{\partial x}+\operatorname{Pr} \nabla^{4} \psi+\operatorname{Pr} \operatorname{Pr} Q\left(\frac{\partial \phi}{\partial z} \frac{\partial}{\partial x} \nabla^{2} \phi\right.
\end{aligned}
$$

$$
\left.-\frac{\partial \phi}{\partial x} \frac{\partial}{\partial z} \nabla^{2} \phi+\frac{\partial}{\partial x} \nabla^{2} \phi\right)
$$

$\frac{\partial \theta}{\partial t}+\frac{\partial \psi}{\partial z} \frac{\partial \theta}{\partial x}-\frac{\partial \psi}{\partial x} \frac{\partial \theta}{\partial z}=R a \frac{\partial \psi}{\partial x}+\nabla^{2} \theta$

$\frac{\partial \phi}{\partial t}+\frac{\partial \psi}{\partial z} \frac{\partial \phi}{\partial x}-\frac{\partial \psi}{\partial x} \frac{\partial \phi}{\partial z}=\frac{\partial \psi}{\partial x}+\operatorname{Pr}_{m} \nabla^{2} \phi$,

where $\psi$ is the streamfunction, $\theta$ is the temperature deviation from the conductive steady state profile, and $\phi$ is the magnetic flux related to the magnetic field $\boldsymbol{B}$ in two dimensions by $\boldsymbol{B}=\hat{\boldsymbol{y}} \times \nabla \phi$, where $\hat{\boldsymbol{y}}$ is a unit vector orthogonal to the $x-z$ plane. Also, $\operatorname{Pr}$ is the Prandtl number, $P r_{m}$ is the magnetic Prandtl number, $R a$ is the Rayleigh number, and $Q$ is the Chandrasekhar number which characterizes the "initial strength of the mean-squared horizontal magnetic field in the region" (Lantz, 1995). (See Lantz and Sudan (1995) for the precise definition of $Q$.) The boundary conditions are similar to those in Sect. 2, plus conducting magnetic boundary conditions for $\phi$; see Lantz (1995) and Lantz and Sudan (1995) for further discussion.

Consider the following Galerkin expansions:

$$
\begin{aligned}
\psi(x, z, t)=A(t) \sin (a x) \sin (z)+B(t) \sin (z) \\
+C(t) \cos (a x) \sin (2 z), \\
\theta(x, z, t)=D(t) \cos (\text { ax }) \sin (z)+E(t) \sin (2 z) \\
+F(t) \sin (a x) \sin (2 z)+K(t) \sin (4 z), \\
\phi(x, z, t)=G(t) \cos (\text { ax }) \sin (z)+H(t) \sin (2 z) \\
+I(t) \sin (\text { ax }) \sin (2 z)+J(t) \sin (4 z) .
\end{aligned}
$$

This is an extension of the original Lantz (1995) model, which did not include the $K \sin (4 z)$ and $J \sin (4 z)$ terms. The addition of the $K \sin (4 z)$ term is required in the temperature expansion to maintain conservation of energy (Thiffeault and Horton, 1996; Gluhovsky and Tong, 1999). The addition of the $J \sin (4 z)$ term in the magnetic flux expansion is required to transform the LOM into a system of coupled gyrostats, to be discussed below. (Note also that the original Lantz model reduces to the HK model when $G \equiv H \equiv I \equiv Q \equiv 0$.) The resulting equations for the modified Lantz model are as follows:

$$
\dot{A}=-\operatorname{Pr}\left(1+a^{2}\right) A+\frac{a P r}{1+a^{2}} D+\frac{a\left(3+a^{2}\right)}{2\left(1+a^{2}\right)} B C
$$




$$
\begin{gathered}
\quad-a \operatorname{Pr} r_{m} \operatorname{Pr} Q\left[\frac{3-a^{2}}{1+a^{2}} G H+G\right], \\
\dot{B}=-\operatorname{Pr} B-\frac{3 a}{4} A C-\operatorname{Pr}_{m} \operatorname{Pr} Q \frac{3 a}{4} G I, \\
\dot{C}=-\operatorname{Pr}\left(4+a^{2}\right) C-\frac{a P r}{4+a^{2}} F-\frac{a^{3}}{2\left(4+a^{2}\right)} A B \\
\quad+a \operatorname{Pr} r_{m} \operatorname{Pr} Q I+2 a \operatorname{Pr} r_{m} \operatorname{Pr} Q \frac{12-a^{2}}{4+a^{2}} I J, \\
\dot{D}=-\left(1+a^{2}\right) D+\operatorname{Ra} a A-a A E-\frac{a}{2} B F, \\
\dot{E}=-4 E+\frac{a}{2} A D, \\
\dot{F}=-\left(4+a^{2}\right) F-\operatorname{Ra} a C+\frac{a}{2} B D+2 a C K, \\
\dot{G}=-\operatorname{Pr}_{m}\left(1+a^{2}\right) G+a A-a A H-\frac{a}{2} B I, \\
\dot{H}=-4 \operatorname{Pr}_{m} H+\frac{a}{2} A G, \\
\dot{I}=-\operatorname{Pr}_{m}\left(4+a^{2}\right) I-a C+\frac{a}{2} B G+2 a C J, \\
\dot{J}=-16 \operatorname{Pr}_{m} J-a C I, \\
\dot{K}=-16 K-a C F .
\end{gathered}
$$

Equation (21) corrects a sign error that appeared in the last term of Eq. (12a) of Lantz (1995) (S. R. Lantz, personal communication). This 11-mode model has a coupled gyrostats form, as shown by the linear change of variables,

$$
\begin{aligned}
A^{\prime} & =\frac{1}{\sqrt{2}} A, \\
B^{\prime} & =\frac{1}{\sqrt{1+a^{2}}} B, \\
C^{\prime} & =\sqrt{\frac{1}{2}\left(\frac{4+a^{2}}{1+a^{2}}\right)} C, \\
D^{\prime} & =D, \\
E^{\prime} & =\sqrt{2} E-\sqrt{2}\left(R a+\frac{P r}{2\left(1+a^{2}\right)}\right), \\
F^{\prime} & =F, \\
G^{\prime} & =\sqrt{\frac{P r_{m} \operatorname{Pr} Q}{2}} G, \\
H^{\prime} & =2 \sqrt{\frac{2 \operatorname{Pr} \operatorname{Pr} Q}{1+a^{2}}} H, \\
I^{\prime} & =\sqrt{\frac{\operatorname{Pr} \operatorname{Pr}_{2}}{2}} \frac{4+a^{2}}{1+a^{2}} I, \\
J^{\prime} & =4 \sqrt{\frac{\operatorname{Pr} \operatorname{Pr} Q}{1+a^{2}}} J, \\
K^{\prime} & =\sqrt{2} K-\frac{1}{\sqrt{2}}\left(\operatorname{Ra}+\frac{P r}{2\left(1+a^{2}\right)}\right) .
\end{aligned}
$$

Dropping primes, the resulting set of equations appears in Table 2.
In Table 2, the parameters have the following definitions:

$$
\begin{aligned}
p_{1} & =a \frac{3+a^{2}}{2 \sqrt{4+a^{2}}}, & p_{3} & =-\frac{3 a}{2} \frac{1}{\sqrt{4+a^{2}}}, \\
q_{1} & =-\frac{3 a}{2 \sqrt{4+a^{2}}}, & q_{3} & =-\frac{a}{2} \frac{1+a^{2}}{\sqrt{4+a^{2}}}, \\
r_{1} & =-\frac{a^{3}}{2 \sqrt{4+a^{2}}}, & r_{3} & =\frac{a}{2} \sqrt{4+a^{2}}, \\
p_{2} & =-\frac{a}{2 \sqrt{2}} \frac{3-a^{2}}{\sqrt{1+a^{2}}}, & p_{4} & =\frac{a}{2} \frac{12-a^{2}}{4+a^{2}} \sqrt{1+a^{2}}, \\
q_{2} & =-\frac{a}{2} \sqrt{\frac{1+a^{2}}{2}}, & q_{4} & =\frac{a}{2} \sqrt{1+a^{2}}, \\
r_{2} & =2 a \sqrt{\frac{2}{1+a^{2}}}, & r_{4} & =-\frac{8 a \sqrt{1+a^{2}}}{4+a^{2}}, \\
k_{1} & =\frac{a P r}{\left(1+a^{2}\right) \sqrt{2}}, & k_{3} & =2 a \sqrt{\frac{1+a^{2}}{4+a^{2}}}, \\
k_{2} & =\frac{a}{2} \sqrt{1+a^{2}}, & k_{4} & =\frac{a P r}{\sqrt{2\left(1+a^{2}\right)\left(4+a^{2}\right)}}, \\
f_{1} & =4 \sqrt{2}\left(\operatorname{Ra}+\frac{P r}{2\left(1+a^{2}\right)}\right), & f_{2} & =\frac{16}{\sqrt{2}}\left(\operatorname{Ra}+\frac{P r}{2\left(1+a^{2}\right)}\right),
\end{aligned}
$$

and $\gamma=\sqrt{\operatorname{Pr} r_{m} \operatorname{Pr} Q}$, and the dissipation parameters are

$$
\begin{array}{ll}
\alpha_{A}=\operatorname{Pr}\left(1+a^{2}\right), & \alpha_{F}=\left(4+a^{2}\right), \\
\alpha_{B}=\operatorname{Pr}, & \alpha_{K}=16, \\
\alpha_{C}=\operatorname{Pr}\left(4+a^{2}\right), & \alpha_{G}=\operatorname{Pr}\left(1+a^{2}\right), \\
\alpha_{D}=\left(1+a^{2}\right), & \alpha_{H}=4 \operatorname{Pr}_{m}, \\
\alpha_{E}=4, & \alpha_{I}=\operatorname{Pr}_{m}\left(4+a^{2}\right), \\
& \alpha_{J}=16 \operatorname{Pr}_{m} .
\end{array}
$$

In Table 2, the friction and (constant) forcing terms appear to the immediate left of the first vertical bar. Following the first vertical bar are seven gyrostats. Gyrostats I (an Euler gyroscope), II (the original Lorenz gyrostat), III (a degenerative gyroscope), and IV (another Lorenz gyrostat) comprise the energy-conserving Thiffeault and Horton (1996) model. Gyrostats V (with three nonlinear and two linear terms), VI (another Euler gyroscope), and VII (another gyrostat with three nonlinear and two linear terms) are all added as the result of the inclusion of the magnetic field. They represent the interaction of the magnetic flux modes with all three streamfunction modes. Note further that the original Lantz (1995) model, missing the $K$ and $J$ terms, cannot be transformed into the form of coupled gyrostats. This leads to potential pathological behavior, as we now discuss.

Consider the original Lantz (1995) model, Eqs. (21-31) but with $J \equiv K \equiv 0$. This system has an invariant manifold,

$A=B=D=E=G=H=0$,

on which the equations for the remaining variables, $C, F$, and $I$, are exactly linear and give unstable solutions for $R a>R a_{c 2}$, where

$R a_{c 2}=\frac{\left(4+a^{2}\right)^{3}}{a^{2}}+Q\left(4+a^{2}\right)$.

Therefore the original Lantz (1995) model has solutions diverging to infinity. Adding the modes $K \sin (4 z)$ and $J \sin (4 z)$ eliminates the existence of the invariant manifold, thanks to the presence of Eqs. (30-31). Moreover, the modified Lantz model's coupled gyrostat structure guarantees 
Table 2. The modified Lantz model in coupled gyrostats form.

$$
\begin{aligned}
& \dot{A}=-\alpha_{A} A \\
& \dot{B}=-\alpha_{B} B \\
& \dot{C}=-\alpha_{C} C \\
& \dot{D}=-\alpha_{D} D \\
& \dot{E}=-\alpha_{E} E-f_{1} \\
& \dot{F}=-\alpha_{F} F \\
& \dot{K}=-\alpha_{K} K-f_{2} \\
& \dot{G}=-\alpha_{G} G \\
& \dot{H}=-\alpha_{H} H \\
& \dot{I}=-\alpha_{I} I \\
& \dot{J}=-\alpha_{J} J \\
& \mid \begin{array}{l}
+p_{1} B C \\
+q_{1} A C
\end{array} \\
& +r_{1} A B \\
& \text { - } a A E \\
& +k_{1} D \\
& -a A E-k_{1} A-k_{2} B F \\
& +a A D \text {, } \\
& +k_{2} B D+k_{3} C K+k_{4} C, \\
& -k_{3} C F \text {, } \\
& \mid \\
& \text { III } \\
& +k_{4} F\left|\begin{array}{|c|}
+p_{2} G H-a \gamma G, \\
+q_{2} A H+p_{3} G I, \\
+r_{2} A G, \\
\mathrm{~V}
\end{array}\right| \begin{array}{r}
+q_{3} B I, \\
+r_{3} B G \\
\mathrm{VI}
\end{array}\left|\begin{array}{c}
+q_{4} C J-a \gamma C, \\
+r_{4} C I, \\
\mathrm{VII}
\end{array}\right|
\end{aligned}
$$

conservation of energy and bounded solutions (Gluhovsky and Tong, 1999). This is explicitly shown by verifying that the sum of squares of all dynamic variables is conserved in the absence of forcing and dissipation, as in any system of coupled gyrostats.

These observations do not cast doubt on Lantz's original work since, like Howard and Krishnamurti (1986), he studied his LOM in regimes which avoided the pathological behavior, and he obtained results consistent with numerical simulations of the full PDEs (Lantz and Sudan, 1995). Nonetheless, the implication of the results reported here is to show how further systematic and physically-motivated extensions of magnetoconvection LOMs could be carried out. A suggestion for future study would be the addition of a $\sin (3 z)$ term in the streamfunction expansion, which in the LOM for external shear was needed for vorticity conservation (see Sect. 2). In fact, an external shear mechanism similar to that of Sect. 2 could also be added to the modified Lantz model.

\section{Conclusions}

In this paper, we show that the HK model can be extended to incorporate either a representation of externally forced vertical shear or magnetoconvection effects with shear, by using the coupled gyrostats framework for designing LOMs. This results in LOMs with modular structures which maintain the conservation properties of interest and avoid having solutions that diverge to infinity, which can result from the usual approach of arbitrarily truncating the Galerkin expansions. Note that unlike the original HK and Lantz models, their coupled gyrostat counterparts have no parameter regimes where solutions diverge to infinity.

The coupled gyrostats approach has also been applied to the study of three-dimensional Rayleigh-Bénard convection (Tong and Gluhovsky, 2002) as well as thermosolutal convection (Gluhovsky, 1982) and thermal convection with rotation (Gluhovsky, 1986; Gluhovsky and Agee, 1997). Furthermore, it is easy to show that the Rucklidge (1992) model of "double convection," which can be used to model Rayleigh-Bénard convection with one of four possible physical mechanisms (solutal diffusion, rotation, or an imposed vertical or horizontal magnetic field), has the form of two coupled gyrostats (a Lorenz gyrostat and a gyrostat with three nonlinear terms and one pair of linear terms) plus friction and forcing.

Moving beyond thermal convection, other hydrodynamic problems lend themselves to the coupled gyrostats approach. For instance, a hierarchy of low-order models for the barotropic, quasigeostrophic potential vorticity equation for a beta plane atmosphere with topography has been shown to have the building block structure of coupled gyrostats (Gluhovsky et al., 2002). Systems of coupled gyrostats can also serve as shell models for turbulence (Gluhovsky and Tong, 1999). Finally, a LOM for the magnetohydrodynamics of a toroidal confinement device (Chen et al., 1990) can easily be shown to have the form of a gyrostat with three nonlinear terms and one pair of linear terms. It is also notable that Hamiltonian LOMs based on gyrostats inherit the Hamiltonian structure (hence all conservation properties) of the original equations (Gluhovsky, 2006). These examples provide further evidence that coupled gyrostats can be employed in a physically motivated, modular approach to designing LOMs based on spectral Galerkin approximations.

\section{Appendix A}

\section{Converting a LOM into coupled gyrostats form}

In Sects. 2 and 3, we use linear changes of variables to convert a system of equations into explicit coupled gyrostats form. Here we discuss an informal approach of how to establish such a transformation when confronted with a candidate LOM. A more general, algorithmic method is given by Lakshmivarahan and Wang (2008a,b). Since we did not learn of their work until recently, we did not use their methodology 
in the work reported here. In the interest of disclosure, we present below how we found our transformations.

Our informal approach begins with attempting to write the original system of equations in an array similar to Tables 1 and 2. The idea is to arrange the nonlinear and linear terms into coupled subsystems, each involving three dynamical variables and a triplet of nonlinear terms. In some cases, such a subsystem may have only two, not three nonlinear terms (e.g. the Lorenz (1963) model). Once all the nonlinear terms are grouped into triplets, next associate every linear term to one of the triplets (those involving the variable in this particular term), except the friction terms. A friction term is a linear term using the same variable that appears on the left hand side of the equation containing it. The friction terms are set off on in a special column right after the equal sign, along with constants (see, e.g. Table 1).

Now, every nonlinear and linear term should belong to a subsystem that looks like one of the special cases of a gyrostat discussed in Gluhovsky and Tong (1999), except for their coefficients and signs. (If not, there will be some remaining "orphan" terms.) If a candidate subsystem looks almost like a gyrostat but is missing a linear term, sometimes such a term may be generated by transforming a variable involved in a nonlinear term. For instance, if there is a nonlinear term $X Y$ and the same row is missing a linear term proportional to $X$, then the transformation $Y=a Y^{\prime}+b, X=X^{\prime}$ will turn the nonlinear term into $a X^{\prime} Y^{\prime}+b X^{\prime}$, producing the missing linear term. In some cases, there is no hope of generating the missing linear term, so that the subsystem may not be a gyrostat after all. The presence of such incomplete triplets or orphan terms may suggest that the system of equations cannot be converted into coupled gyrostat form. (The inability of a LOM to be convertible into such a form may often be verified by identifying an invariant manifold on which the LOM is linear and, in certain regimes, possesses unstable solutions.)

If there are no such pathologies, the final step is to convert the coefficients and signs of the terms in the subsystems to explicitly match the pattern of Eqs. (1). This is done by writing a general linear change of variables, e.g., for the $i$ th variable, let $Y_{i}=a_{i} Y_{i}^{\prime}+b_{i}$, where $a_{i}$ and $b_{i}$ are arbitrary unknowns. Some of these unknown constants are constrained by the condition that they need to be used to generate missing linear terms as described above. Another constraint comes from the requirement not to generate new linear terms where they are unnecessary, again because the relevant variable is involved in a nonlinear term.

After executing the transformations, and setting the resulting coefficients equal to those required by coupled gyrostat form, one obtains a system of algebraic equations for the $a_{i}$ 's and $b_{i}$ 's with more unknowns than equations. This indicates that the required transformation is non-unique. From here, by trial and error one can establish a particular transformation that is valid. For instance, one can successively set some of the $a_{i}$ 's and $b_{i}$ 's to 1 and 0 , respectively, until the number of unknowns equals the number of equations, then solve for the remaining unknowns. While desirable, simplicity can be elusive, as shown by the examples in Sects. 2 and 3.

Acknowledgements. We are grateful to E. M. Agee and S. Lakshmivarahan for their encouragement, and S. R. Lantz for valuable correspondence regarding his work. We also thank S. R. Lantz, $\mathrm{K}$. Azer, and the referees for comments that considerably improved the manuscript. A. G. was supported by NSF grants ATM-0514674 and ATM-0541491.

Edited by: H. A. Dijkstra

Reviewed by: S. Lakshmivarahan and two other anonymous referees

\section{References}

Agee, E. M.: Mesoscale cellular convection over the oceans, Dyn. Atmos. Oceans, 10, 317-341, 1987.

Atkinson, B. W. and Zhang, J. W.: Mesoscale shallow convection in the atmosphere, Rev. Geophys., 34, 403-431, 1996.

Bénard, H. and Avsec, D.: Travaux récents sur les tourbillons cellulaires et les tourbillons en bandes: applications à l'astrophysique et à la météorologie, J. Phys. Radium, 9, 486-500, 1938.

Brown, R. A.: Longitudinal instabilities and secondary flows in the planetary boundary layer: a review, Rev. Geophys. Space Phys., 18, 683-697, 1980.

Brunt, D.: Experimental cloud formation, in: Compendium of Meteorology, Malone, T. F. (Ed.), American Meteorological Society, Boston, 1255-1262, 1951.

Chandra, K.: Instability of fluids heated from below, Proc. R. Soc. Lon. Ser.-A, 164, 231-242, 1938.

Chandrasekhar, S.: Hydrodynamic and Hydromagnetic Stability, Clarendon Press, Oxford, 1961.

Chen, H., Shan, X., and Montgomery, D.: Galerkin approximations for dissipative magnetohydrodynamics, Phys. Rev. A, 42, 61586165, 1990.

Chen, Z.-M. and Price, W. G.: Chaotic behavior of a Galerkin model of a two-dimensional flow, Chaos, 14, 1056-1068, 2004.

Chen, Z.-M. and Price, W. G.: On the relation between RayleighBénard convection and Lorenz system, Chaos Soliton. Fract., 28, 571-578, 2006

Fütterer, C.: Growth of nonlinear patterns in binary-fluid convection, analysis of models, Theor. Comp. Fluid Dyn., 16, 467-478, 2003.

Getling, A. V.: Rayleigh-Bénard Convection: Structures and Dynamics, World Scientific, Singapore, 1998.

Gluhovsky, A. B.: Nonlinear systems that are superpositions of gyrostats, Sov. Phys. Dokl., 27, 823-825, 1982.

Gluhovsky, A. B.: On systems of coupled gyrostats in problems of geophysical hydrodynamics, Izv. Acad. Sci. USSR Atmos. Oceanic Phys., 22, 543-549, 1986.

Gluhovsky, A.: Energy-conserving and Hamiltonian low-order models in geophysical fluid dynamics, Nonlin. Processes Geophys., 13, 125-133, 2006, http://www.nonlin-processes-geophys.net/13/125/2006/.

Gluhovsky, A. and Agee, E.: An interpretation of atmospheric loworder models, J. Atmos. Sci., 54, 768-773, 1997. 
Gluhovsky, A. and Tong, C.: The structure of energy conserving low-order models, Phys. Fluids., 11, 334-343, 1999.

Gluhovsky, A., Tong, C., and Agee, E.: Selection of modes in convective low-order models, J. Atmos. Sci., 59, 1383-1393, 2002.

Graham, A.: Shear patterns in an unstable layer of air, Philos. T. R. Soc. A, 232, 285-296, 1933.

Hermiz, K. B., Guzdar, P. N., and Finn, J. M.: Improved loworder model for shear flow driven by Rayleigh-Bénard convection, Phys. Rev. E, 51, 325-331, 1995.

Holmes, P., Lumley, J. L., and Berkooz, G.: Turbulence, Coherent Structures, Dynamical Systems, and Symmetry, Cambridge University Press, Cambridge, 1996.

Howard, L. N. and Krishnamurti, R.: Large scale flow in turbulent convection: a mathematical model, J. Fluid Mech., 170, 385410, 1986.

Ingersoll, A. P.: Convective instabilities in plane Couette flow, Phys. Fluids, 9, 682-689, 1966.

Kelly, R. E.: The onset and development of thermal convection in fully developed shear flows, Adv. Appl. Mech., 31, 35-112, 1994.

Koschmieder, E. L.: Bénard Cells and Taylor Vortices, Cambridge University Press, Cambridge, 1993.

Krishnamurti, R. and Howard, L. N.: Large-scale flow generation in turbulent convection, Proc. Natl. Acad. Sci. USA, 78, 19811985, 1981.

Lakshmivarahan, S. and Y. Wang: On the structure of the energy conserving low-order models and their relation to Volterra gyrostat, Nonlinear Analysis: Real World Applications, in press, 2008a.

Lakshmivarahan, S. and Y. Wang: On the relation between energy conserving low-order models and a system of coupled generalized Volterra gyrostats with nonlinear feedback, J. Nonlinear Sci., 18, 75-97, 2008b.

Lakshmivarahan, S., Baldwin, M. E., and Zheng, T.: Further analysis of Lorenz's maximum simplification equation, J. Atmos. Sci., 63, 2673-2699, 2006.

Lantz, S. R.: Magnetoconvection dynamics in a stratified layer, II. A low-order model of tilting instability, Astrophys. J., 441, 925941, 1995.

Lantz, S. R. and Sudan, R. N.: Magnetoconvection dynamics in a stratified layer, I. Two-dimensional simulations and visualization, Astrophys. J., 441, 903-924, 1995.
Lorenz, E. N.: Deterministic nonperiodic flow, J. Atmos. Sci., 20, 130-141, 1963.

Moehlis, J., Faisst, H., and Eckhardt, B.: A low-dimensional model for turbulent shear flows, New J. Phys., 6, 56, 17 pp., 2004.

Mutabazi, I., Wesfreid, J. E., and Guyon, E. (Eds.): Dynamics of Spatio-Temporal Cellular Structures: Henri Bénard Centenary Review, Springer, New York, 2006.

Obukhov, A. M.: Integral invariants in hydrodynamic systems, Sov. Phys. Dokl., 14, 32-35, 1969.

Peltier, W. R. (Ed.): Mantle Convection, Gordon and Breach, New York, 1989.

Peng, X. D., Tang, C. J., and Qiu, X. M.: Minimal dynamic model for electron-temperature-gradient turbulence, Phys. Plasmas, 14, 0107034 pp., 2007.

Proctor, M. R. E. and Weiss, N. O.: Magnetoconvection, Rep. Prog. Phys., 45, 1317-1379, 1982.

Roy, D. and Musielak, Z. E.: Generalized Lorenz models and their routes to chaos. III. Energy-conserving horizontal and vertical mode truncations, Chaos Soliton. Fract., 33, 1064-1070, 2007.

Rucklidge, A. M.: Chaos in models of double convection, J. Fluid Mech., 237, 209-229, 1992.

Saltzman, B.: Finite amplitude free convection as an initial value problem-I, J. Atmos. Sci., 19, 329-341, 1962.

Shirer, H. N.: On cloud street development in three dimensions: parallel and Rayleigh instabilities, Beitr. Phys. Atmos., 59, 126149, 1986.

Shirer, H. N. (Ed.): Nonlinear Hydrodynamic Modeling: A Mathematical Introduction, Springer, Berlin, 1987.

Thiffeault, J. L. and Horton, W.: Energy-conserving truncations for convection with shear flow, Phys. Fluids, 8, 1715-1719, 1996.

Tong, C. and Gluhovsky, A.: Energy-conserving low-order models for three-dimensional Rayleigh-Bénard convection, Phys. Rev. E, 65, 046306, 11 pp., 2002.

Treve, Y. M. and Manley, O. P.: Energy conserving Galerkin approximations for 2-D hydrodynamic and MHD Bénard convection, Physica D, 4, 319-342, 1982.

Volterra, V.: Sur la théorie des variations des latitudes, Acta Math., 22, 201-358, 1899. 